\title{
Management of Intracranial Incidental Findings on Brain MRI
}

\section{Management intrakranieller Zufallsbefunde in der MRT-Bildgebung}

Authors

Affiliations
S. Langner ${ }^{1}$, R. Buelow ${ }^{1}$, S. Fleck² ${ }^{2}$ A. Angermaier ${ }^{3}$, M. Kirsch ${ }^{1}$

Institute for Diagnostic Radiology and Neuroradiology, Universitymedicine Greifswald, Germany

Department of Neurosurgery, Universitymedicine Greifswald, Germany

Department of Neurology, Universitymedicine Greifswald, Germany
Key words

- incidental finding

- brain

- MRI received $\quad 7.2 .2016$

accepted $\quad 30.5 .2016$

\section{Bibliography}

DOI http://dx.doi.org/

10.1055/s-0042-111075

Published online: 19.7.2016

Fortschr Röntgenstr 2016; 188 :

1123-1133 @ Georg Thieme

Verlag KG Stuttgart · New York .

ISSN 1438-9029

\section{Correspondence}

\section{PD Dr. Sönke Langner}

Institut für Diagnostische Radiologie und Neuroradiologie, Universitätsmedizin Greifswald Ferdinand-Sauerbruch-Str. 1

17475 Greifswald

Germany

Tel.: ++ 49/3834/866960

Fax: ++ 49/38 34/86 7097

soenke.langner@uni-greifswald. de

\section{Abstract \\ $\nabla$}

The wider use of MRI for imaging of the head in both research and clinical practice has led to an increasing number of intracranial incidental findings. Most of these findings have no immediate medical consequences. Nevertheless, knowledge of common intracranial incidental findings and their clinical relevance is necessary to adequately discuss the findings with the patient. Based on the author's experiences from a large populationbased study, the most common incidental MR findings in the brain will be presented, discussing their clinical relevance and giving recommendations for management according to the current literature and guidelines.

Key points:

- Intracranial incidental findings are common.

- The majority of these findings have no immediate medical consequences.

- Knowledge of common incidental findings is necessary for appropriate management.

Citation Format:

- Langner S, Buelow R, Fleck S et al. Management of Intracranial Incidental Findings on Brain MRI. Fortschr Röntgenstr 2016; 188: 1123-1133

\section{Zusammenfassung \\ $\nabla$}

Durch eine Zunahme von MR-Untersuchungen des Kopfes, sowohl im Rahmen von Studien als auch in der klinischen Routine, kommt es zu einer steigenden Anzahl intrakranieller Zufallsbefunde. Die Mehrzahl dieser Befunde hat keine unmittelbare medizinische Konsequenz. Dennoch sind Kenntnisse über häufige intrakranielle Zufallsbefunde und ihre klinische Relevanz notwendig, um den Befund adäquat mit dem Patienten diskutie- ren zu können. Basierend auf den eigenen Erfahrungen aus einer populationsbasierten MR-Studie sowie fMRT-Studien werden die häufigsten intrakraniellen Zufallsbefunde in der MRT dargestellt und ihre klinische Relevanz bzw. Empfehlungen zum Management entsprechend der aktuellen Literatur und Leitlinien aufgezeigt.

\section{Introduction}

$\nabla$

Nowadays, continuous technical improvement in MR systems, the sequences used as well as the widespread use of high-field technology have resulted in ability of MRI to morphologically detect even minor changes in the brain. The number of MR examinations performed as part of the clinical routine has increased due to the high soft-tissue contrast without the use of radiation. Furthermore, magnetic resonance imaging is an integral part of population-based studies, such as the "Study of Health in Pomerania" (SHIP) [1] the study of the German national cohort [2] or the Rotterdam Study [3]. In addition, there has been an increase in the number of functional MRI examinations performed in the course of neurological studies. Consequently an increasing number of incidental findings have been detected. An incidental finding is a previously unrecognized abnormality with potentially clinical relevance that has been detected by chance, and not related to the basis for the examination [4]. The number and frequency of incidental findings are dependent on the examination modality [5] such as the field strength or sequence used as well as the patient or volunteer cohort $[1,6]$. The frequency of intracerebral incidental findings described in the literature ranges between $2-32 \%$. However, the majority of these findings did not 
require further medical clarification $[1,3,6]$, yet they represent an ethical and practical challenge, since they are unsettling to the patient or volunteer while having potential medical, financial or lifestyle consequences [7].

Patients expect the examining radiologist to provide a medical explanation as well as advice [8]. Therefore familiarity with frequently-occurring incidental findings and their various clinical consequences is important when adequately discussing the findings with the patient and, if necessary, recommending further consultation with relevant specialists. In clinical practice, such knowledge reduces the risk of overlooking a finding or misdiagnosing it [9]. In addition, the majority of volunteer subjects of neuroscientific studies have expected to be informed of possible existing abnormalities [10]. Although there is still no national or international consensus regarding responding to incidental findings [10], current population-based MR studies $[1,2]$ have established an internally standardized approach to incidental findings which in addition to categorizing findings, also particularly regulates communication of such findings as well as any required clarification, since this is likewise of importance $[1,2,10]$.

This review article is based on the authors' experience with a population-based MR study [1] as well as 12 years' experience performing neurological functional MRI studies, and presents intracranial incidental findings frequently encountered during MRI, as well as their clinical relevance. The article also provides recommendations for management based on current literature as well as guidelines of the relevant individual professional associations.

\section{Classification of Incidental Findings}

Based on previous studies [1 -3] and taking into account ethical aspects $[1,4]$, incidental findings can be assigned to various categories with respect to their clinical relevance and resulting consequences. In this overview, the classification system of two large population-based MR studies [1,2] is used to classify incidental findings as: normal variations, incidental findings without clearly defined diagnostic consequences (Category I, "non-reportable"); findings requiring additional medical clarification (Category II, "reportable") and findings requiring emergency clarification (Category III, "actionable"). 0 Table 1 provides an overview of the classification of incidental findings into their respective categories. Depending on severity, assignment to different categories is possible. This will be discussed individually in the relevant sections. In our experience as well as in the literature, Category III generally comprises the least number of findings $[1,3,6]$. Unless otherwise indicated, the prevalence described in the individual sections is based on the prevalence in a normal healthy population as indicated in the literature.

\section{Literature Review}

Recommendations in this review article for management of individual incidental findings reflect the established approach in large population-based MR studies [1, 2]. In addition a Medline review for the 2002-2016 time frame was performed using the key words "incidental findings", "brain MRI", "management", "population-based imaging", "prevalence”, "guidelines", “ventricular system", "intracranial cyst", "pineal gland cyst", "cerebral microbleeds", "white matter hyperintensities", "radiological isolated syndrome", "intracranial aneurysm", "intracranial vascular malformation", "intracranial stenosis", "incidental stroke", "meningioma", "incidental glioma", "pituitary adenoma". Both primary literature and supplementary secondary literature for the respective findings were included. A further research of the guidelines of the relevant professional associations via the online portal www.awmf.org was performed using the respective incidental finding as a search term (last access 20 January 2016). These guidelines were assessed with respect to their recommendations for clinical management.

Table 1 Classification of individual incidental findings into the different categories.

\begin{tabular}{|c|c|c|}
\hline category I & category II & category III \\
\hline ventricular system variations & pineal cysts & \\
\hline arachnoidal cysts & colloid cysts & \\
\hline \multicolumn{3}{|l|}{ pineal cysts } \\
\hline \multicolumn{3}{|l|}{ enlarged perivascular spaces } \\
\hline \multicolumn{3}{|l|}{ choroid plexus cysts } \\
\hline cerebral microhemorrhages, solitary & cerebral microhemorrhages, multiple & $\begin{array}{l}\text { intracranial macrohemorrhages, intraparencymatous/ } \\
\text { extracerebral }\end{array}$ \\
\hline \multirow[t]{2}{*}{ WMH (Fazekas Grade I) } & WMH (Fazekas Grade II/III) & acute diffusion impairment \\
\hline & radiological isolated syndrome & \\
\hline intracranial stenosis $<50 \%$ & $\begin{array}{l}\text { intracranial stenosis }>50 \% \text { or pronounced WMH } \\
\text { (Fazekas Grade II/III) }\end{array}$ & \\
\hline $\begin{array}{l}\text { cavernoma in non-eloquent area } \\
\text { without indication of hemorrhage } \\
\text { and/or older patient/volunteer }\end{array}$ & $\begin{array}{l}\text { cavernoma in other location or indication of } \\
\text { hemorrhage or younger patient/volunteer }\end{array}$ & \\
\hline developmental venous anomaly (DVA) & intracranial aneurysm & \\
\hline capillary telangiectasia & arteriovenous malformation (AVM) & \\
\hline \multirow[t]{3}{*}{$\begin{array}{l}\text { meningioma, calcified/no perifocal } \\
\text { edema/older patient/volunteer }\end{array}$} & $\begin{array}{l}\text { meningioma with perifocal edema/younger } \\
\text { patient/hyperintense signal in } T 2 w\end{array}$ & \\
\hline & $\begin{array}{l}\text { endogenous brain tumor without contrast } \\
\text { accumulation }\end{array}$ & endogenous brain tumor with contrast accumulation \\
\hline & pituitary mass (micro/macroadenoma) & \\
\hline
\end{tabular}




\section{Examination Protocol}

Unlike clinical practice, neuroscientific studies are particularly characterized by a limited number of structural sequences [10]. Moreover, modification of the examination protocol in the course of studies is frequently not possible $[1,2]$, as this could make the differential diagnosis of incidental findings more difficult. Therefore recommendations for additional MR sequences or other radiological examination methods to confirm the findings are provided in the respective sections.

\section{Ventricular System}

A normal variation of the interventricular septum ( $\bullet$ Fig. 1) is frequently observed. A distinction can be made between a cavum vergae, a cavum veli interpositum and a cavum septum pellucidum; the latter is the most common variant [11]. In all weightings, the changes appear as fluid-isointense in the MRI. Even though associations with various neurological disorders have been described [12], such changes do not require further clarification (Category I).

Between $6-10 \%$ of all examinations exhibit limited asymmetry of the ventricular system ( $\bullet$ Fig. 1 ) not requiring further clarification (Category I) [13]; in such cases the right side is emphasized. The literature does not indicate clearly defined thresholds with respect to the degree of tolerated asymmetry. Pronounced asymmetry can be indicative of a possible drainage obstruction. In such cases, a possible structural lesion in the region of the interventricular foramen should be ruled out, and if needed a neurosurgical work-up should be performed (Category II).

In the differential diagnosis, normal pressure hydrocephalus should be considered if dilated temporal horns and rounded posterior horns are present with prominent ventricles in disproportion to narrow CSF spaces above the hemispheres ( $\bullet$ Fig. $\mathbf{1}$ ), and if there is no indication of an obstruction in the region of the interventricular foramen or aqueduct [14]. This is primarily a clinical diagnosis with a typical symptom triad (so-called Hakim triad) consisting of incontinence, gait disturbance and mental decline. Since this is a treatable form of dementia [14], further clarification should be performed (Category II) if this is suspected. If imaging raises suspicion of hydrocephalus, additional axial T2-weighted (T2w) or FLAIR images should be acquired to identify possible pressure caps. Further, the examination protocol should include high-resolution sagittal T2w images (e.g. CISS) of the aqueduct in order to rule out an obstruction.

\section{Intracranial Cysts \\ $\nabla$}

\section{Arachnoidal Cysts}

These are the most common cystic intracranial anomaly with a prevalence of $0.3-1.4 \%[1,3]$. They are caused by a duplication or diverticulum of the arachnoid in an early phase of development [15] and are typically located in the region of the cisterns or above the hemisphere ( $\bullet$ Fig. 2 ). Despite their local space consuming effect on the brain, the literature does not provide any indication of association with clinical symptoms [15], so that - apart from very large cysts - further clarification is not necessary (Category I). Diffusion-weighted imaging (DWI) in which the epidermoid cyst appears hyperintense is suited to distinguish from the main differential diagnosis of epidermoid cyst.

\section{Pineal Cysts}

Cystic changes in the pineal gland are a common incidental finding, with a prevalence of up to $10 \%[16,17]$. As a rule they have a diameter $<1 \mathrm{~cm}$, but can reach a diameter of up to $2 \mathrm{~cm}$ ( $\bullet$ Fig. 2). Septa and solid components can be demonstrated in uncomplicated cysts, and calcification can result from regressive changes. As a rule, the cysts appear minimally hyperintense in T1-weighted (T1w) and T2w images; there is no signal suppression in FLAIR. In high-resolution T2w images (e.g. CISS) cysts exhibit a low hypointense signal. Small pineal cysts are clinically irrelevant (Category I). Larger cysts can compress both the tectal plate and aqueduct, thus leading to headache [18]. No threshold has been defined in the literature; thus in the case of clinical symptoms, a neurosurgical work-up is called for (Category II). Purely morphologically, it is frequently not possi-
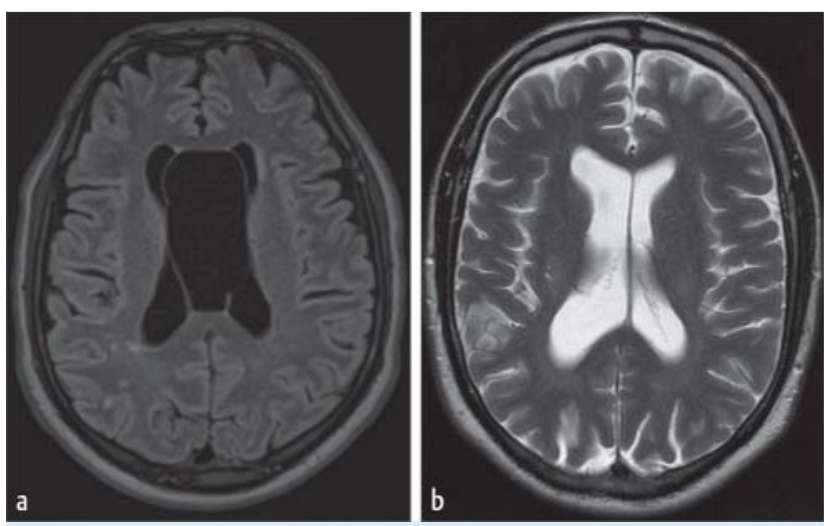

Fig. 1 Incidental findings of the ventricular system. a Axial FLAIR image of a 23-year old fMRI volunteer demonstrates a Cavum septi pellucidi et Vergae with a doubling of the interventricular septum. $\mathbf{b}$ Axial T2w image of a 27-year old patient with migraine. Asymmetry of the ventricular system. This is typically located on the right side. c Axial FLAIR image of a 67-year
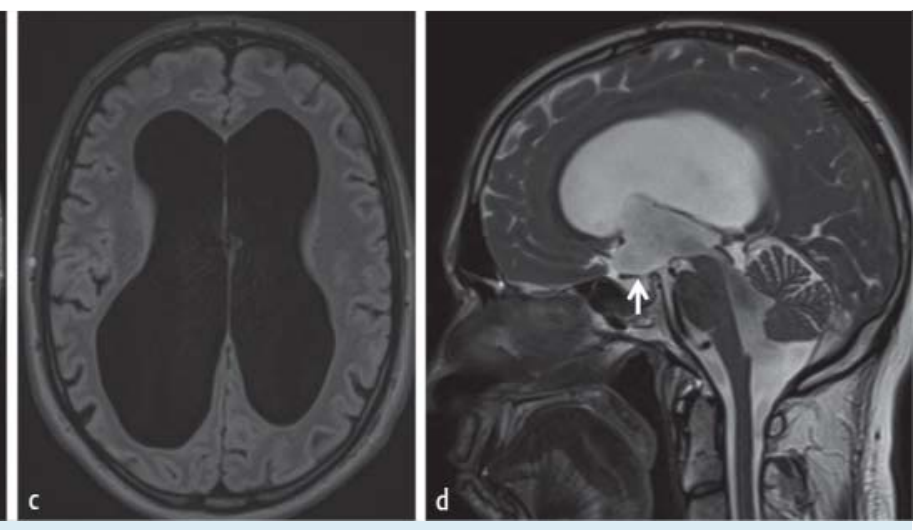

old fMRI volunteer. Symmetrical enlargement of the ventricular system and a mismatch between inner and outer CSF spaces. $\mathbf{d}$ Sagittal T2w image of the same volunteer as in c demonstrating downward bowing of the floor of the third ventricle indicating hydrocephalus. The volunteer was referred for further neurosurgical work-up. 

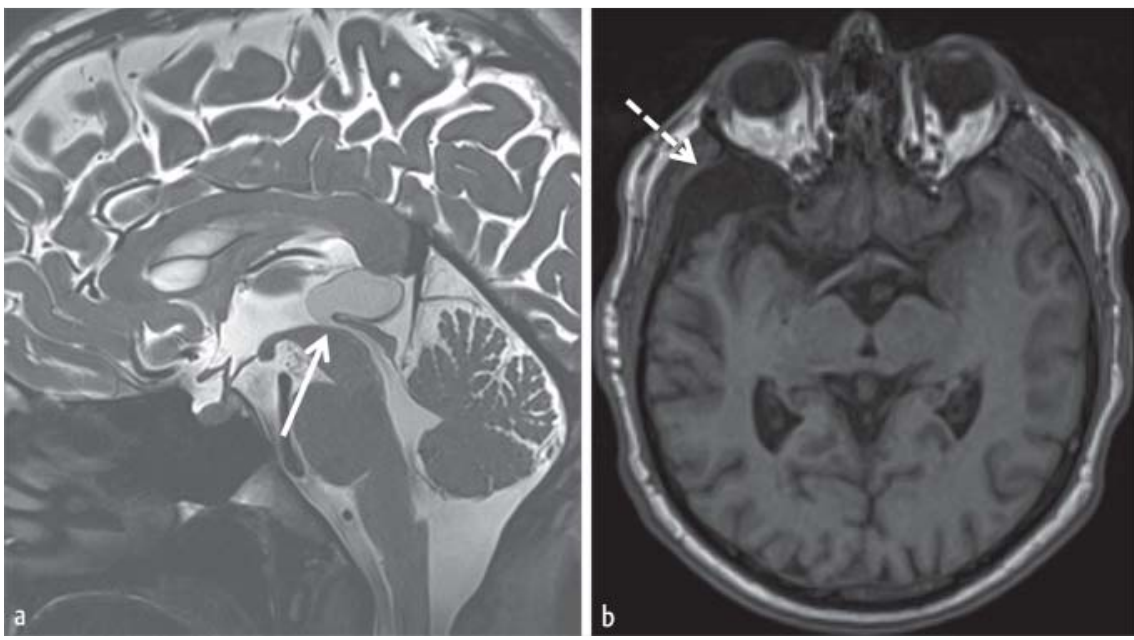

Fig. 2 Cystic intracranial incidental findings. a Sagittal T2w image of a 25-year old fMRI volunteer. Large pineal gland cyst with hypointense signal. The cyst is bulging into the aqueduct. Due to the history of headache in the mornings with associated vomitting, the patient was referred for further neurosurgical work-up. b Axial T1w image of 32-year old female patient. The MRI was performed for the exclusion of Multiple Sclerosis. Right temporopolar arachnoid cyst (dotted arrow) with local space occupying effect but no associated perifocal edema.

ble to distinguish it from a pinealcytoma in the image [17]. In the case of highly inhomogeneous cysts, further examination using contrast and a follow-up shortly thereafter is recommended.

\section{Enlarged Perivascular Spaces}

Enlarged perivascular spaces (also called Virchow-Robin spaces (VR)) are interstitial fluid-filled areas surrounding the penetrating medullary arteries and adjoining the subarachnoid space. There is no consensus regarding the definition of "enlarged"; the majority of authors indicate a threshold of 2-3 mm [19]. It should be noted that VR spaces can be delineated using $3 \mathrm{~T}$ and are typically located in the basal ganglia in the region of the anterior perforated substance as well as in the white matter of the cerebrum. As a rule they are small, but can occupy substantial space (๑ Fig.3). Unlike lacunar ischemic lesions, VR spaces are liquor-isointense in all sequences and do not exhibit signal alteration in the adjoining medullary layer in FLAIR images. Although expanded VR spaces can be indicative of a number of neurological disorders [19], normally they reflect a change that does not require further clarification (Category I).

\section{Choroid Plexus Cysts}

These are non-neoplastic, non-inflammatory cysts within the choroid plexus; they are usually bilateral and multicentric. The cysts mainly appear isointense in T1 $\mathrm{w}$ images, in T2w they are minimally hyperintense and strongly hyperintense in DWI ( $\bullet$ Fig.3). They are clinically irrelevant (Category I), requiring no further clarification [20].

\section{Colloid Cysts}

Colloid cysts are protein-rich cysts on the roof of the third ventricle lying closely to the interventricular foramen (๑ Fig.3). Typically these cysts appear hypointense in T1w, and hyperintense in $\mathrm{T} 2 \mathrm{w}$; the signal correlates with the consistency of the cyst content. Although this is a rare incidental finding $[3,17]$, such cysts should be referred to a neurosurgeon (Category II), since hydrocephalus may result from a blockage of the interventricular foramen [21]. If there is sufficient suspicion, additional high-resolution
T2w images (e.g. CISS) should be acquired in order to assess the positional relationship to the roof of the third ventricle and the interventricular foramen. Contrast-enhanced sequences are generally not required. Frequently colloid cysts can be easily identified in CCT as hyperdense lesions.

\section{Intracranial Hemorrhages \\ $\nabla$}

\section{Macrohemorrhages}

As a rule, intracranial hemorrhaging is symptomatic (Category III), particularly in cases of intraparenchymal hemorrhage. Since signal behavior of the hemorrhage is agedependent, and hemorrhages appear isointense in the hyperacute ( $<12$ hours) and acute (12 hours to 2 days) stages in native T1w images frequently used in neurological studies, additional CT diagnosis should be performed if there is corresponding suspicion. In rare cases, however, large subdural hematomas or hygromas can be asymptomatic [1]. There is no data in the literature regarding the prevalence of asymptomatic subdural hematomas [1, 3, 6]. Typically they are concavely located above the hemisphere. Chronic subdural hematomas typically exhibit a hypointense signal in $\mathrm{T} 1 \mathrm{w}$ and $\mathrm{T} 2 \mathrm{w}$ images as well as in T2* $\mathrm{w}$ gradient echo (GRE) images. In FLAIR and DWI the changes appear hyperintense as a rule ( $\bullet$ Fig. 4$)$. Since surgery is the therapy of choice particularly in cases of large hematomas [22], a neurosurgeon should be consulted immediately (Category III).

\section{Microhemorrhages}

Cerebral microhemorrhages (cerebral microbleeds, CBM) are intracerebral petechial hemorrhages that appear as punctate signal drop-outs in T2* $\mathrm{w}$ GRE images or SWI (susceptibility-weighted imaging) sequences ( $\bullet$ Fig.4). In the literature the prevalence ranges from $4.5-9.6 \%$ [23, $24]$; prevalence is dependent on the age of the patient as well as the examination technology used. Thin-slice SWI sequences with $3 \mathrm{~T}$ disclose more lesions that thick-slice GRE sequences with $1.5 \mathrm{~T}$. Detection of a single lesion has limited diagnostic value [24]; evidence of at least two 

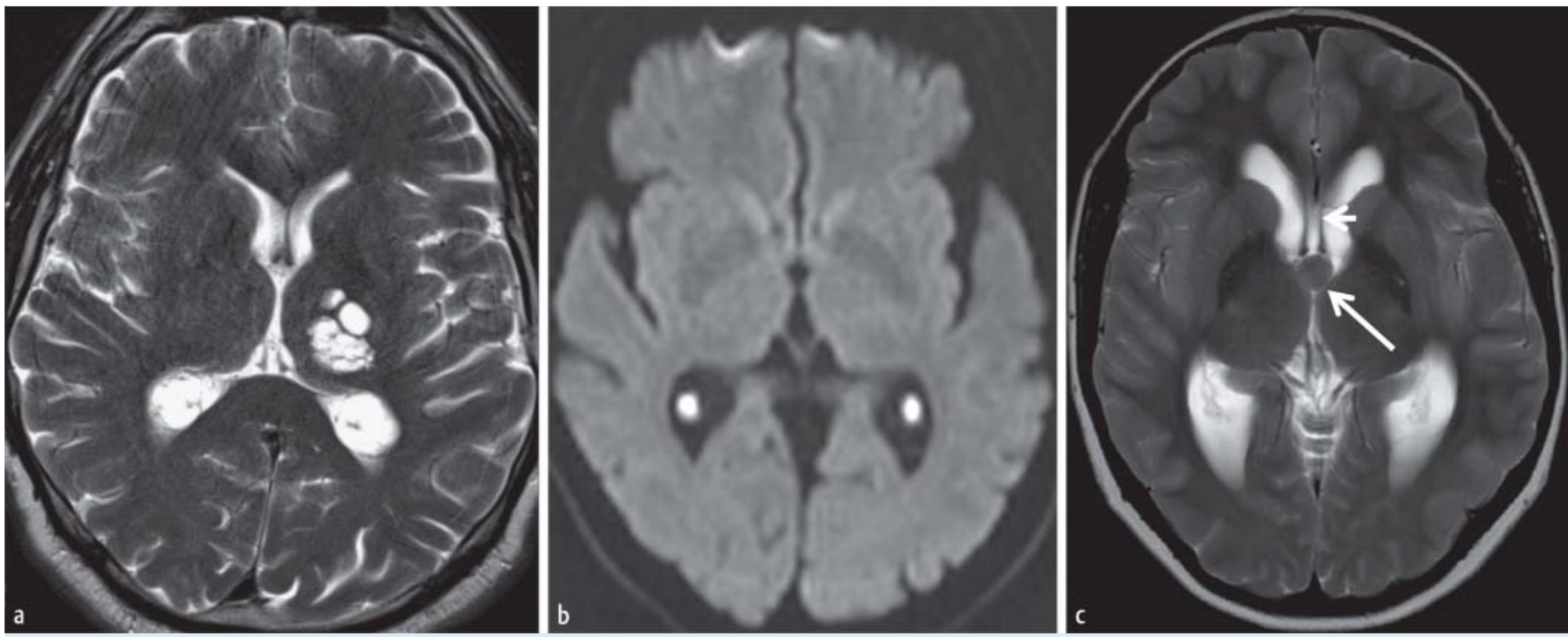

Fig. 3 Cystic intracranial incidental findings. a Axial T2w image of a 42-year female patient with headache. Enlarged perivascular spaces in the thalamus of the left side. These lesions are isointens to CSF in all sequences. $\mathbf{b}$ Axial diffusionweighted images $(b=1000 \mathrm{~s} / \mathrm{mm} 2)$ of a 53 -year old female patient. The MRI was performed to exclude intracranial pathologies causally related to vertigo. Hyperintens cysts of the choroid plexus on both sides (cholester- olcysts). c Axial T2w image of a 25-year old fMRI volunteer. Colloid cyst at the roof of the third ventricle (large arrow) with close relationship to the Foramen of Monroi on both sides. The cyst is hypointens to CSF. Due to the risk of obstructive hydrocephalus the patient was referred for further neurosurgical work-up.
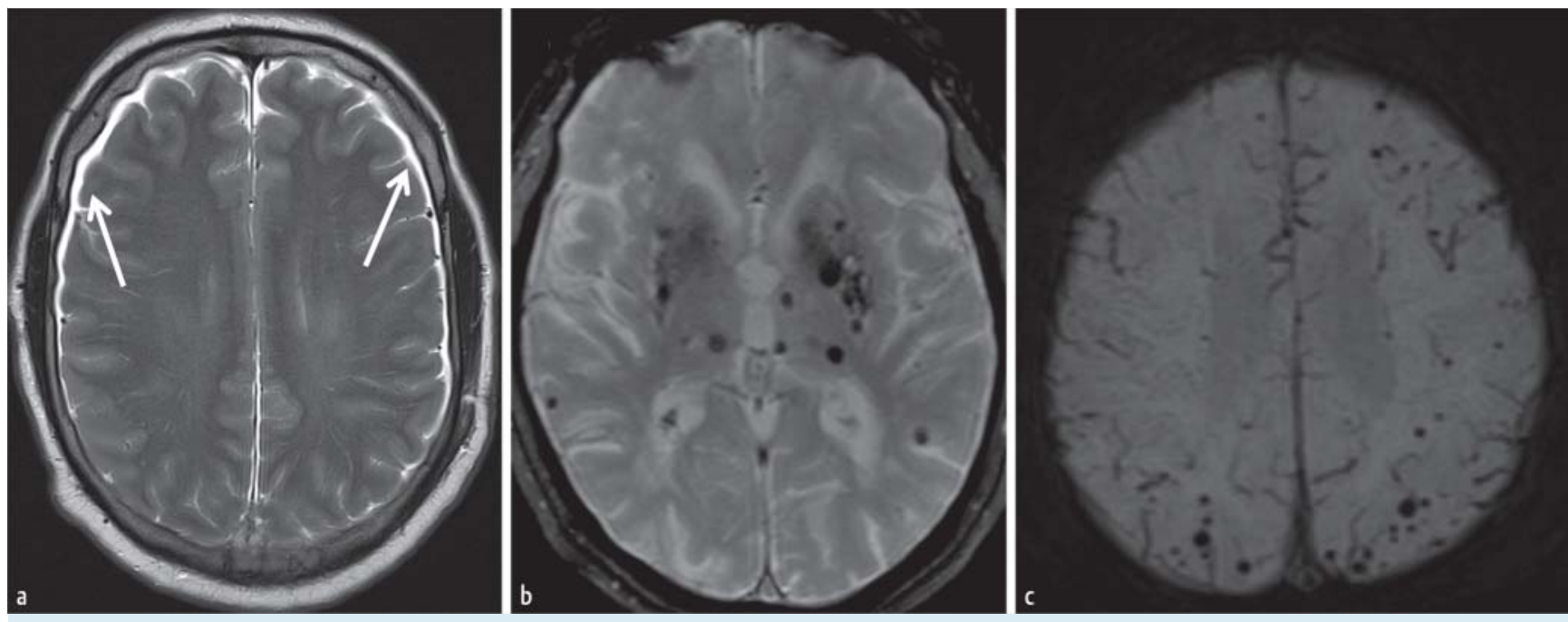

Fig. 4 Incidental intracranial hemorrhages. a Axial T2w image of a 19-year old fMRI volunteer. Small subacute subdural hematomas (arrow) at both hemispheres. Also there was no space occupying effect, the patient was referred for neurosurgical work-up. b Axial T2*w image of a 47-year old patient. The MRI was performed due to headache. Multiple hypointens lesions in the basal ganglia on both sides as a typical finding of hypertensive encephalopathy. The patient was referred for optimization of cardio-vascular risk factors. c Axial SWI image (minIP reconstruction) of 72-year old female patient. The MRI was performed due to vertigo. Multiple peripherally located signal voids indicative of microhemorrhages due to cerebral amyloid angiopathy (CAA; histologically proven). lesions should be assessed as "positive" [23, 24]. In the majority of cases cerebral microbleeds are asymptomatic, but gain critical relevance if there are multiple occurrences. Thus the presence of multiple CBMs in otherwise healthy patients is a possible predictor of a cerebral vascular event [24]; when correlated with the extent of cognitive dysfunction, they can be indicative of an underlying disorder, depending on their distribution [24] (० Fig.4). Furthermore, there should be an additional investigation into cardiovascular risk factors if there are multiple CBMs (Category II).

\section{Changes in White Matter \\ $\nabla$}

\section{Leukoaraiosis}

Leukoaraiosis (white matter hyperintensities, $\mathrm{WMH}$ ) is a descriptive term for rarefication of the white matter 
caused by damage to the medullary layer arteries [25]. In T2w and Flair images, these changes appear as patchy or flat increases in signal which typically omit the subcortical $\mathrm{U}$ fibers ( $\bullet$ Fig. 5 ). The prevalence of $\mathrm{WMH}$ increases with age $[3,26]$, and the changes are more pronounced in patients with cardiovascular risk factors and symptomatic cerebrovascular diseases. White matter hyperintensities can be quantified automatically or semiquantified visually [26]. Visual semiquantification can be performed in routine clinical practice. The Fazekas scale is an established means of classifying according to three levels of severity: Grade I: mild WMH, individual punctate lesions < $10 \mathrm{~mm}$; Grade II: moderate WMH; individual lesions between 10-20mm; Grade III: severe WMH, confluent lesions; individual or confluent lesions $>20 \mathrm{~mm}$ [27]. In addition, a current meta-analysis has described a relationship between the extent of $\mathrm{WMH}$ and the risk of stroke, cognitive dysfunction and development of dementia [26]. Therefore, in cases of extensive WMH (Fazekas Grade II/II), there should be further investigation (Category II) with respect to cardiovascular risk factors $[25,28]$.

\section{Radiological Isolated Syndrome}

Hyperintense lesions detected in $\mathrm{T} 2 \mathrm{w}$ or FLAIR images which in shape, size and location ( $\bullet$ Fig.5) appear like demyelinating masses in multiple sclerosis in otherwise neurological persons are characterized as radiological isolated syndrome (RIS) [29]. Prevalence among young people $15-24$ years of age is $0.1 \%$ [30]. Within the following $2.3-5.4$ years, approx. $40 \%$ of these patients develop neurological symptoms, and $10 \%$ will formally meet the diagnostic criteria for multiple sclerosis [31]. Detected asymptomatic myelon lesions increases the risk of developing symptoms [32]. As a rule, patients who eventually develop a neurological deficit also exhibit a progression of lesions in T2w and FLAIR images. Although there is no current consensus regarding the type and extent of possible therapy [32], a neurological work-up should be performed (Category II).
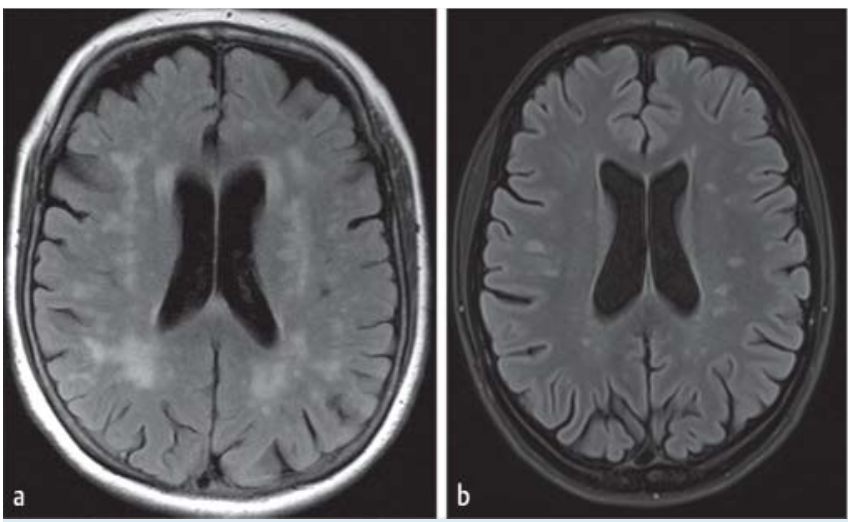

Fig. 5 Incidental white matter lesions. a Axial FLAIR image of a 54-year old female patient. The MRI was performed to exclude intracranial pathologies related to sinusitis while otherwise no previous medical history. Multiple white matter hyperintensities (WMH). Due to the extensive character of the lesions the patient was referred for optimization of cardiovascular risk factors. b Axial FLAIR image of a 23-year old female fMRI volunteer. Multiple hyperintens ovoid lesions. c Sagittal T2w image of the same volunteer as in b. The lesions also affect the corpus callosum (arrow) with involvement of

\section{Vascular Changes}

$\nabla$

\section{Intracranial Stenosis}

Although intracranial atherosclerosis is one of the most common causes of stroke [33], the literature does not provide a uniform statement regarding the prevalence of stenosis. Stenoses are not reliably detected on anatomical sequences. Time of flight (TOF) angiography is superior to contrast-enhanced MRI with respect to local resolution imaging of intracranial vessels. It should be kept in mind, however, that due to the method, stenoses appear exaggerated in TOF angiography. Opinions regarding the management of intracranial stenoses are varied [34]. There should be further clarification of the stenosis (Category II) if the stenosis is $>50 \%$ or extensive $\mathrm{WMH}$ is ascertained (Fazekas Grade II/III) [35], since stenosis progression or the risk of a cerebrovascular event is raised [33].

\section{Stroke}

A stroke is the occurrence of clinical symptoms caused by an ischemic lesion. A distinction should be made regarding clinically silent infarcts (acute incidental infarcts, AII) [36]. The prevalence of AII infarcts lies between $8-28 \%$ [36] and increases with age. In DWI they appear as hyperintense lesions with corresponding signal reduction on the ADC card ( $\bullet$ Fig. 5). In T2w and FLAIR images they are likewise hyperintense, in T1 $w$ images they appear hypointense. After about 3 days subacute infarcts can exhibit barrier disturbance. Most cases of AII are not asymptomatic, but the symptoms described by the patient are not perceived as a stroke by relatives; consequently there is no medical follow-up [37]. Since both a transient ischemic attack (TIA) with a DWI-positive lesion as well as an AII increase the risk of a subsequent stroke [36], they represent an incidental finding requiring immediate investigation (Category III).

\section{Aneurysms}

The prevalence of intracranial aneurysm in the normal adult population lies between $1-7 \%$ [8]; in population-
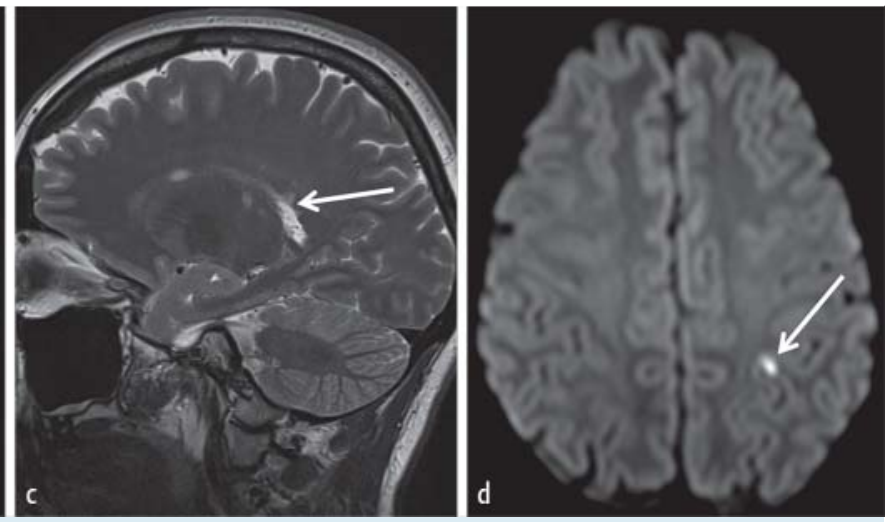

the lower surface (so called "dawson fingers“ or "Hahnenkamm-Phänomen“). The criterias of dissimination in space for Multiple sclerosis are fulfilled. Two years later the patient developed optic neuritis. $\mathbf{d}$ Axial diffusion weighted image $(b=1000 \mathrm{~s} / \mathrm{mm} 2)$ of a 30 -year old female patient with sinusitis. Cortical lacunar acute ischemia postcentrally on the left side (arrow). The patient was immediately referred to the neurological emergency department. 
based MR studies, the prevalence is $1-3 \%[1,3,6]$. Most cases involve a saccular aneurysm of the basal cerebral arteries. The rupture of an intracranial intradural aneurysm, distal to the origin of the ophthalmic artery for the internal carotid artery or distal to the origin of the posterior inferior cerebellar artery for the vertebral artery results in a subarachnoid hemorrhage. Aneurysms can be easily identified if the examination protocol includes MR angiography. Due to its local resolution, TOF angiography also has high sensitivity for even small aneurysms [38]. However, they can also be distinguished in contrast-enhanced T1w images ( 0 Fig. 6 ). The signal in T2w and FLAIR images depends on the flow and possible thrombus within the aneurysm. Opinions regarding the natural progression and risk of rupture of an incidental asymptomatic aneurysm are varied $[8,39,40]$. The individual risk of rupture depends upon a number of factors that cannot be influenced (e.g. size and location of the aneurysm), as well as those that can (e.g. smoking, high blood pressure). Although previously an average rupture risk of $5 \%$ in 5 years was assumed [8], a more recent meta-analysis was able to develop an improved individual score for the initial risk assessment [41]. Due to continuously improved treatment options, a work-up (Category II) of this type of incidental finding should be performed at a neurovascular center regardless of the age of the patient or aneurysm configuration $[8,40]$.

\section{Cavernomas}

Cavernomas make up approx. $10-15 \%$ of all intracranial vascular malformations [39] with a prevalence of approx. $0.6 \%$. These are low flow malformations consisting of multiple thin-walled capillaries without any intervening brain tissue ( $\bullet$ Fig. 7). Thrombi can form within these capillaries. The adjoining medullary layer contains hemosiderin deposits and gliosis. Cavernomas may be grouped freely, can occur sporadically or postradiogenically, and in the majority of cases are located supratentorially. Depending on the location, typical symptoms are headache, seizures, focalneurological deficits or intracerebral hemorrhaging; $20 \%$ of cases remain asymptomatic, however [42]. In T2* w GRE or SWI images, cavernomas appear as signal drop-outs caused by susceptibility artifacts of blood breakdown products of thrombi within the lesion. Thus the artifact is larger than the lesion itself (so-called "blooming" phenomenon). However, T2w images better display the "popcorn-like" character of the changes with an inhomogeneous central core and a marginal hypointense rim. In addition, possible siderosis in the adjacent medulla is a clear indication of previous bleeding. With small lesions in particular, differentiation from microhemorrhages can be difficult. Incidental cavernomas can be monitored (Category II) [39]; surgery is indicated in cases that cannot be managed through medication, or in cases of neurological deficit or MR indications of recurrent hemorrhage in an eloquent region of the brain, or initial hemorrhage in a non-eloquent region, as well as in younger patients (Category II). Management of brain stem cavernomas is controversial; prompt surgical treatment [39] should be considered (Category II).

\section{Developmental Venous Anomaly (DVA)}

Developmental venous anomaly (DVA) [43] is the most common vascular cerebral malformation with a prevalence of $2.6 \%$ in autopsy studies. These are not malformations in the strictest sense, but rather a variant of physiological venous drainage of the brain, in which multiple small parenchymal veins collect in a large collecting vein which can extend either cortically or subependymally [43]. Contrastenhanced $\mathrm{T} 1 \mathrm{w}$ images reveal a typical palm tree sign ( $\odot$ Fig. 8). In particular the collecting vein can be distinguished as a signal drop-out in T2w images. A DVA is asymptomatic and does not bleed. However, in $20 \%$ of cases, it is associated with a cavernoma with a bleeding risk (see previous section). Further investigation is not necessary (Category I).

\section{Capillary Telangiectasias}

Capillary telangiectasias make up approx. $15-20 \%$ of all intracranial vascular malformations [44] with a prevalence of approx. $0.7 \%$. These are a vascular malformation made up of thin-walled capillaries with intervening normal cer-

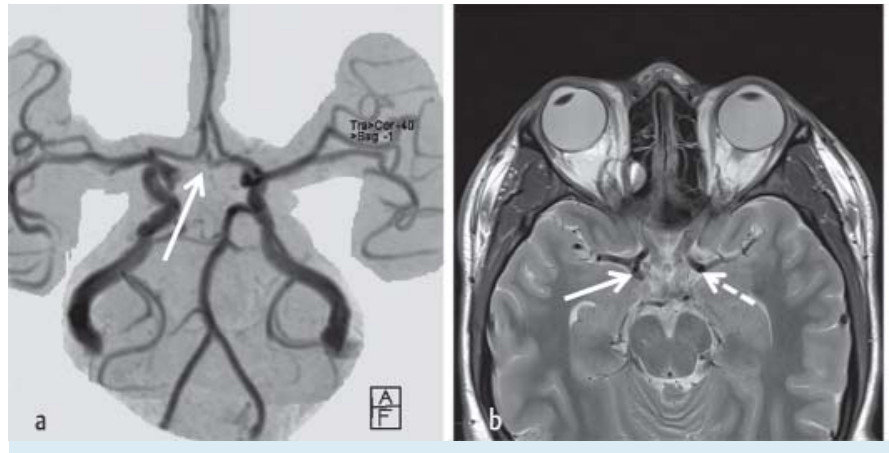

Fig. 6 Incidental intracranial aneurysms. In all the cases the patients and volunteers, respectively, were referred to a neurovascular center due to the size, age or growth tendency of the aneurysm. a Inverted MIP-reconstruction of a TOF-MRA. 45-year old fMRI-volunteer with a small aneurysm of the anterior communicating artery (arrow). b Axial T2w image of a 23-year old patient with non-specific vertigo. The aneurysm at the origin of the posterior communicating artery on the right side (arrow) causes a flow void

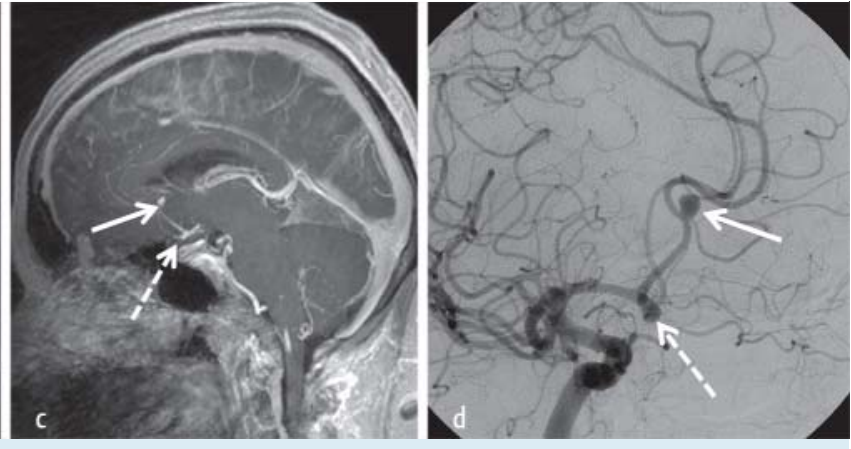

(unaffected carotid-T on the contralateral side, dotted arrow). c Sagittal contrast-enhanced T1w image of a 45-year old female patient with Multiple Sclerosis. The MRI was performed as an annual control. Detection of two new grown aneurysms of the anterior cerebral artery (arrow) and the anterior communicating artery (dotted arrow). d Intraarterial catheter angiograpy (XA) of the internal carotid artery of the same patient as in c demonstrating the good correlation between MRI and XA. 

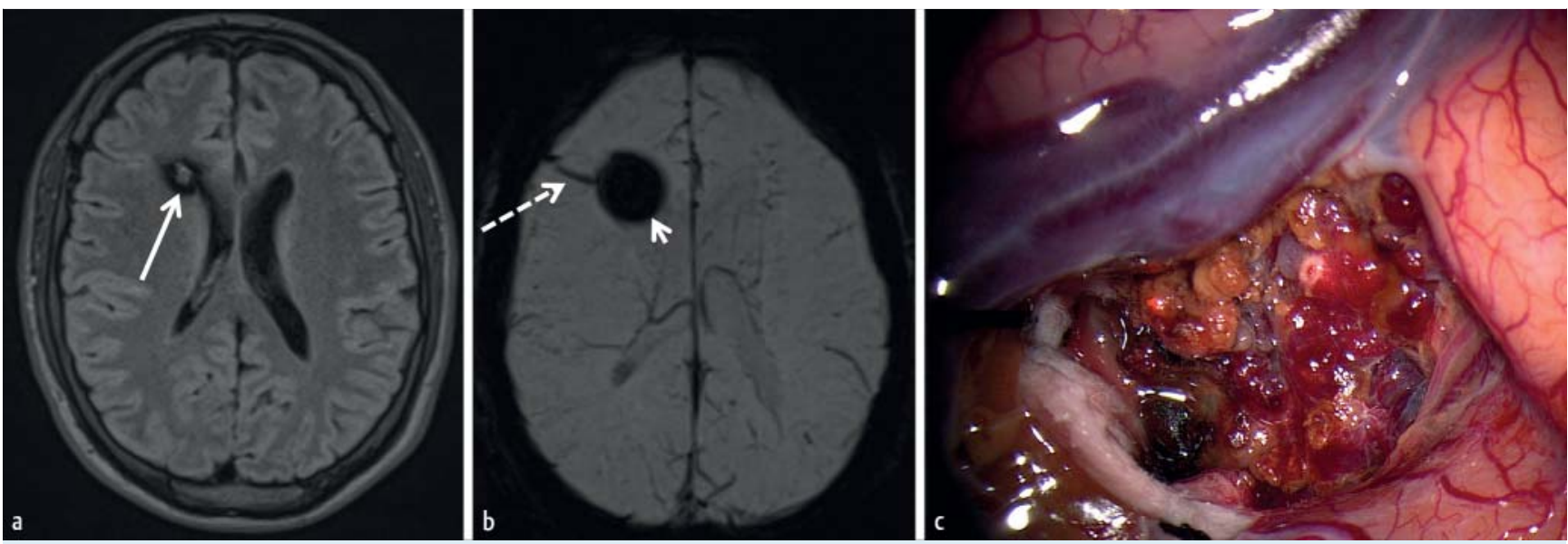

Fig. 7 Cavernomas. a Axial FLAIR image of a 47-year old fMRI volunteer. Inhomogenous lesion in the right frontal white matter with surrounding hypointens siderosis (arrow). Due to the siderosis as an indicator for repeated hemorrhages in non-eloquent areas the patient was referred for further neurosurgical work-up. b Axial SWI image (minIP reconstruction) of the same patient as in a. The cavernoma (arrow head) increases in size (blooming phenomenon). The collecting draining vein appears as tubular hypointens structure. $\mathbf{c}$ Intraoperative picture of the same patient as in a demonstrating the „popcorn“-like appearance of the lesion. ebral parenchyma. They are a relatively common incidental finding, mainly located in the region of the pons and generally asymptomatic. In FLAIR images capillary telangiectasias appear low-contrasting hyperintense; in $\mathrm{T} 2 * \mathrm{~W}$ and SWI images they exhibit significant signal reduction. Administration of contrast agent results in low-contrast accumulation ( $\bullet$ Fig. 8). Typically these changes are shown on only 1-2 slices. Further clarification is unnecessary (Category I), since these are benign lesions [43, 44].

\section{Arteriovenous Malformations (AVM)}

In clinical practice, the term "arteriovenous malformation" is frequently a synonym for a pial AVM, i.e. a short-circuit connection between arteries and veins supplying the brain. Although AVM is the most frequent cause of an intracerebral hemorrhage in young adults, its prevalence is minor at $0.1 \%[39,45]$. In $50 \%$ of cases, an AVM is manifested by intracerebral hemorrhaging; other common symptoms include headaches, seizures or a focal-neurological deficit. The pathological short-circuit results in dilation of the arterial feeder vessels and draining veins. In T2w images these blood vessels appear as tubular signal void artifacts that after administration of contrast agent demonstrate definite accumulation ( $\bullet$ Fig. 8). An AVM is classified according to its size, location and type of venous drainage [46]. The individual risk of hemorrhage depends on a number of factors, including location, drainage via the deep brain veins and the presence of an aneurysm in the feeder vessels [39]. Although a current study has demonstrated the superiority of conservative treatment of asymptomatic AVM [47], individual treatment remains a subject of discussion; therefore an examination in a neurovascular center is recommended (Category II).

\section{Intracranial Tumors}

$\nabla$

\section{Meningiomas}

Meningiomas are tumors arising from the meninges and are benign in the majority of cases. Prevalence is about $0.5 \%[1,3,6,48]$. As a rule, in T1w images meningiomas appear iso- or limited hypointense compared to the cortex ( $\bullet$ Fig.9). In $\mathrm{T} 2 \mathrm{w}$ the signal is variable, but hyperintense as a rule. After administration of contrast agent, strong homogeneous accumulation appears in the image, depending on the extent of calcification. Likewise the adjacent dura shows thickening as well as significant accumulation, the so-called "dura tail". The extra-axial location of the tumor outside the cerebral parenchyma can be particularly assessed on $\mathrm{T} 2 \mathrm{w}$ images showing the cortical veins lying between the brain surface and the tumor as tubular signal voids. Therefore in case of related suspicion, contrast-enhanced T1w images should be acquired. Calcifications can be distinguished in $\mathrm{T} 2{ }^{*} \mathrm{w}$ GRE as well as T2w simages. Frequently these changes can be better evaluated in native CCT. Over the course of time, the majority of incidental meningiomas exhibit no or limited growth tendencies [48]. Calcification and an isointense signal to the cortex on T2w images correlate with limited growth, whereas the absence of calcifications, a hyperintense signal in $\mathrm{T} 2 \mathrm{w}$, perifocal edema as well as young patient age are predictive of tumor growth [48]. The treatment is dependent on the location of the tumor. Surgery is indicated for younger patients if there is pronounced perifocal edema, large non-calcified tumors, penetration into the orbit as well as tumors the continued growth of which would make complete resection difficult. In such cases, a neurosurgeon should be consulted (Category II); otherwise a follow-up in 6-12 months can be performed to evaluate the growth tendency of the tumor (Category II).

\section{Endogenous Brain Tumors}

The prevalence of incidental gliomas is much lower than that of meningiomas, and is given as $0.05 \%$ in the literature 

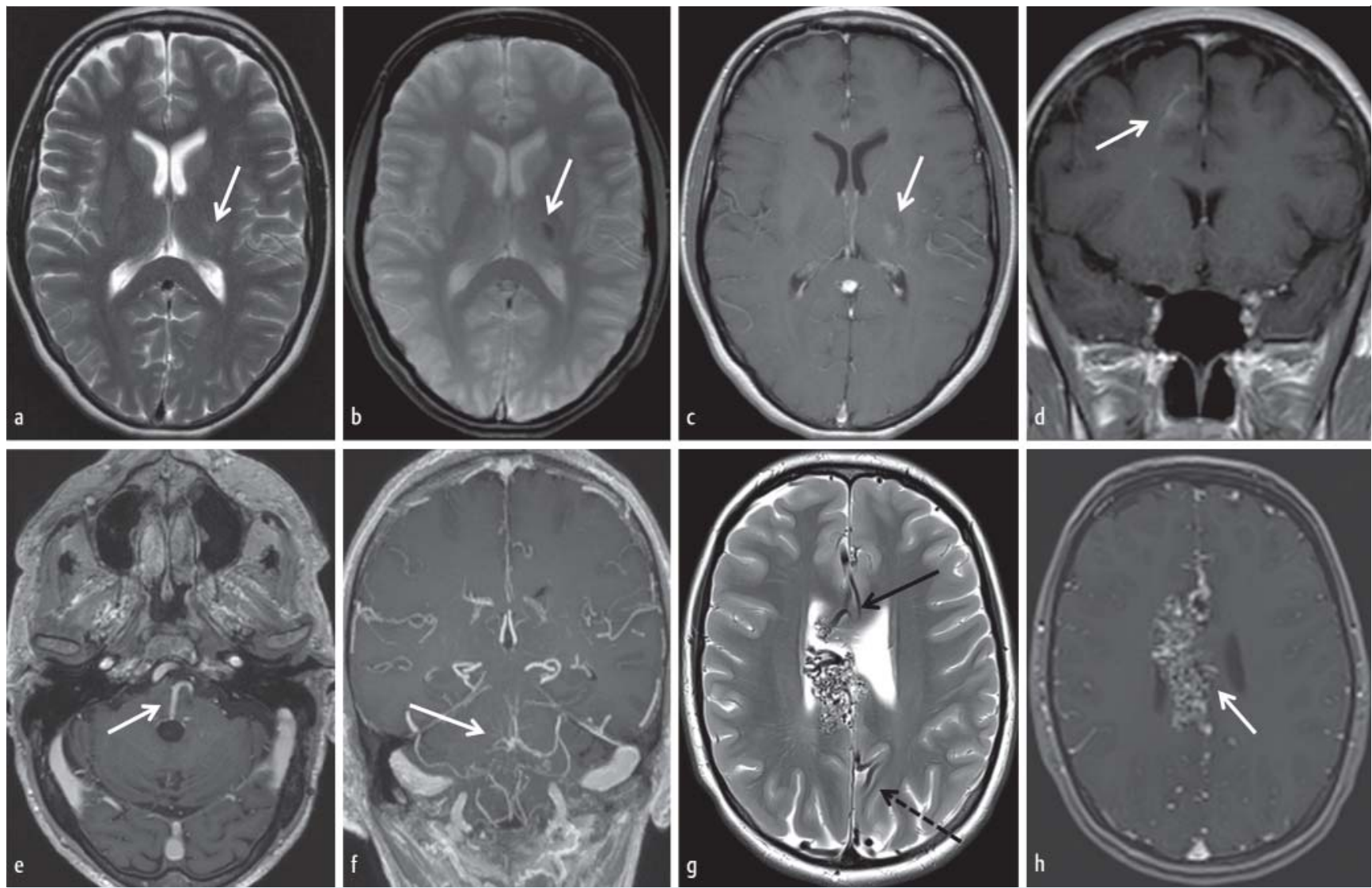

Fig. 8 Vascular Malformations. a Axial T2w image of a 38-year old patient. The MRI was performed due to headache. Typically, capillary teleangiectasie (arrow) appears hyperintens on FLAIR on one slide. $\mathbf{b}$ Axial T2* $w$ image of the same patient as in $\mathbf{a}$ is demonstrating a homogeneous hypointens lesion. The signal loss in capillary teleangiectasia is related to slow flow. c Axial contrast enhanced T1w image of the same patient as in a. Typically, capillary teleangiectasia demonstrates faint enhancement of the lesion. d Contrast enhanced T1w image of a 34-year old female patient. The DVA (arrow) appears as caput medusae due to the confluens of multiple parenchymal veins into one transparenchymal draining vein. e Axial contrast

enhanced T1w image of a 26-year old female patient. Avidly enhancing transparenchymal vein of the DVA within the pons (arrow). $f$ Coronal contrast enhanced T1w image of the same patient as in e) demonstrating the Caput medusae appearance of the lesion. g Axial T2w image of a 23-year old female fMRI volunteer. The dilated arteries (arrow) of the pial AVM of the corpus callosum and the dilated draining vein (dotted arrow) appear as flow voids. $\mathbf{h}$ Contrast enhanced T1 $w$ images of the same patient as in $\mathrm{g}$ ) demonstrating avid enhancement of the vessels and improves the assessment of the angioarchitecture of the lesion.

[49]. In T1w, tumors typically exhibit a hypointense signal ( $\bullet$ Fig. 9); in T2w and FLAIR images they appear as hyperintense with an unclear boundary. In every suspected case, the examination protocol should therefore be supplemented by contrast-enhanced T1w images on at least two spatial planes. As an alternative T1w $3 \mathrm{D}$ data sets (e.g. MPRage) with multiplane reformation are available. Contrast agent accumulation is found only in high-grade endogenous brain tumors which, as a rule, are also symptomatic [49]. Whereas a contrast-enhancing tumor requires further clarification (Category III), opinions vary as to the management of incidental low-grade gliomas [49]; therefore a neuro-oncological work-up should be performed early (Category II).

\section{Pituitary Tumors}

The prevalence of an incidental tumor of the pituitary, a so-called incidentaloma, is indicated to be $0.1 \%$. In the majority of cases these are adenomas, which according to their size can be classified as microadenomas $(<10 \mathrm{~mm})$ and macroadenomas ( $>10 \mathrm{~mm}$ ) ( $\bullet$ Fig. 9 ). As a rule, these

tumors appear isointense compared to the healthy pituitary in T2w and native T1w images. Consequently they can be distinguished either according to their mass (macroadenomas) or low contrast accumulation compared to healthy pituitary tissue (microadenomas). Thus microadenomas represent an incidental finding only in contrast-enhanced images. It should be noted that approx. $35-50 \%$ of all patients up to age 35 exhibit an upward convex pituitary gland. Except for pregnant and nursing women the maximum craniocaudal diameter should not be greater than $10 \mathrm{~mm}$ in sagittal cross-section images [50]. Since microadenomas may be endocrine disruptors, and macroadenomas can cause compression of the optic chiasm due to their size, further endocrinological and neuro-ophthalmological investigation should be performed (Category II) even in cases of low growth tendency [50]. 

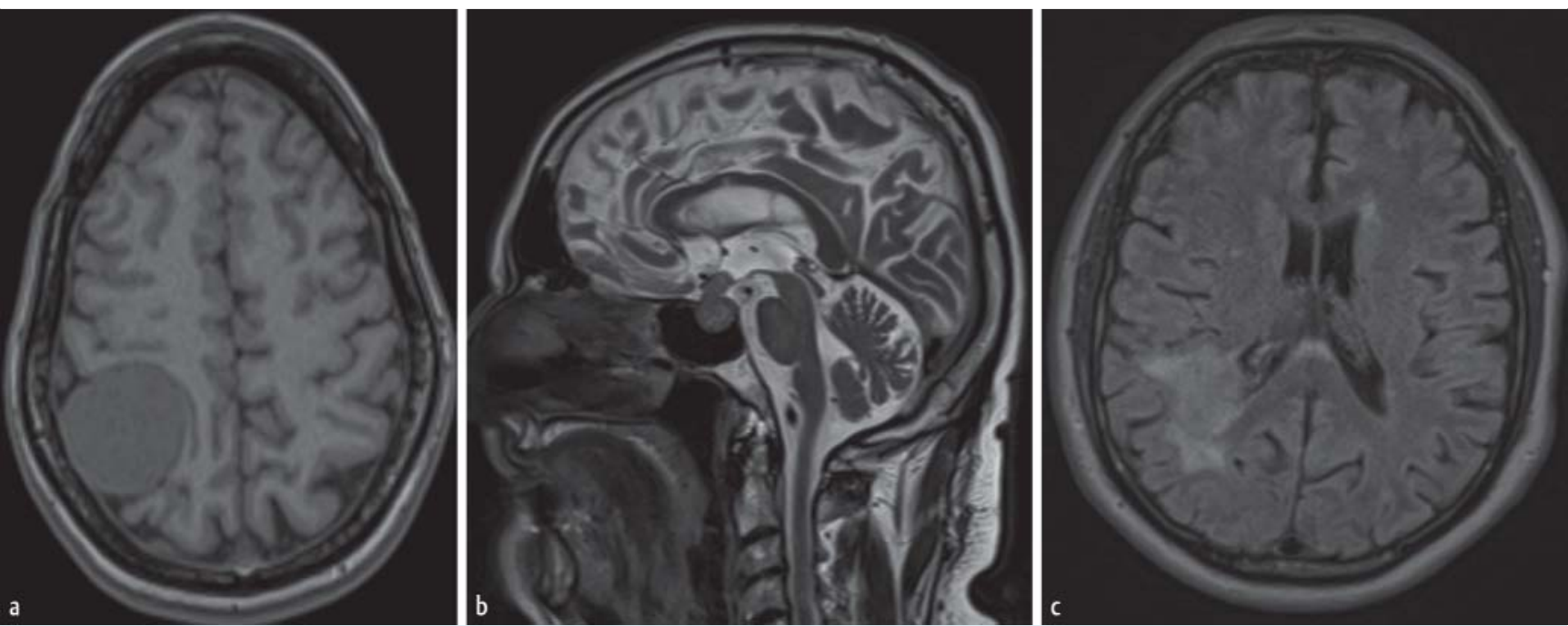

Fig.9 Intracranial incidental tumors. a Axial T1w image of a 32-year old female fMRI volunteer. Postcentrally on the right side located homogenously hypointens meningeoma. Due to their age the patient was referred for further neurosurgical work-up. b Sagittal T2w image of a 53-year old female patient with known Multiple Sclerosis. Beside the demyelinating lesion in the corpus callosum (short arrow) there is tumor with suprasellar growth with central constriction typically for hypophyseal macroadenoma. c Axial FLAIR image of a 22-year old fMRI-volunteer. Right parietal ill defined lesion with discrete mass effect. The patient was referred for neurosurgical work-up. There was a tumor growth on follow-up imaging, therefore the patient underwent stereotactical biopsy (diffuse astrocytoma, WHO ${ }^{\circ} \mathrm{II}$ ).

\section{Summary}

$\nabla$

Intracranial incidental findings are common, but in the majority of cases, they have no immediate medical consequences. Familiarity with common incidental findings, their clinical relevance and recommended management is required in order to discuss the findings adequately with the patient and to initiate further investigation if necessary.

\section{References}

1 Hegenscheid K, Seipel R, Schmidt CO et al. Potentially relevant incidental findings on research whole-body MRI in the general adult population: frequencies and management. European radiology 2013; 23: 816-826

2 Bamberg F, Kauczor HU, Weckbach S et al. Whole-Body MR Imaging in the German National Cohort: Rationale, Design, and Technical Background. Radiology 2015; 277: 206-220

3 Vernooij MW, Ikram MA, Tanghe HL et al. Incidental findings on brain MRI in the general population. The New England journal of medicine 2007; 357: $1821-1828$

4 Illes J, Kirschen MP, Edwards E et al. Ethics. Incidental findings in brain imaging research. Science (New York, NY) 2006; 311: 783-784

5 Orme NM, Fletcher JG, Siddiki HA et al. Incidental findings in imaging research: evaluating incidence, benefit, and burden. Archives of internal medicine 2010; 170: 1525-1532

6 Sandeman EM, Hernandez Mdel C, Morris Z et al. Incidental findings on brain MR imaging in older community-dwelling subjects are common but serious medical consequences are rare: a cohort study. PloS one 2013; 8: e71467

7 Schmidt CO, Hegenscheid K, Erdmann P et al. Psychosocial consequences and severity of disclosed incidental findings from whole-body MRI in a general population study. European radiology 2013; 23: 1343-1351

8 Fiehler J. Unruptured brain aneurysms: when to screen and when to treat? Fortschr Röntgenstr 2012; 184: 97-104

9 Caranci F, Tedeschi E, Leone G et al. Errors in neuroradiology. La Radiologia medica 2015; 120: 795-801

10 Bunnik EM, Vernooij MW. Incidental findings in population imaging revisited. European journal of epidemiology 2016; 31: 1-4

11 Tubbs RS, Krishnamurthy S, Verma K et al. Cavum velum interpositum, cavum septum pellucidum, and cavum vergae: a review. Child's ner- vous system: ChNS: official journal of the International Society for Pediatric Neurosurgery 2011; 27: 1927-1930

12 Trzesniak C, Oliveira IR, Kempton MJ et al. Are cavum septum pellucidum abnormalities more common in schizophrenia spectrum disorders? A systematic review and meta-analysis. Schizophrenia research 2011; 125: $1-12$

13 Kiroglu Y, Karabulut N, Oncel C et al. Cerebral lateral ventricular asymmetry on CT: how much asymmetry is representing pathology? Surgical and radiologic anatomy 2008; 30: 249-255

14 Tarnaris A, Kitchen ND, Watkins $L D$. Noninvasive biomarkers in normal pressure hydrocephalus: evidence for the role of neuroimaging. Journal of neurosurgery 2009; 110: $837-851$

15 Eidlitz-Markus T, Zeharia A, Cohen YH et al. Characteristics and management of arachnoid cyst in the pediatric headache clinic setting. Headache 2014; 54: 1583-1590

16 Berhouma M, Ni H, Delabar V et al. Update on the management of pineal cysts: Case series and a review of the literature. Neuro-Chirurgie 2015; 61: $201-207$

17 Gur RE, Kaltman D, Melhem ER et al. Incidental findings in youths volunteering for brain MRI research. American journal of neuroradiology 2013; 34: 2021 - 2025

18 Langner S, Kirsch M. Radiological Diagnosis and Differential Diagnosis of Headache. Fortschr Röntgenstr 2015; 187: 879-891

19 Potter GM, Doubal FN, Jackson CA et al. Enlarged perivascular spaces and cerebral small vessel disease. International journal of stroke: official journal of the International Stroke Society 2015; 10: 376-381

20 Cakir B, Karakas HM, Unlu E et al. Asymptomatic choroid plexus cysts in the lateral ventricles: an incidental finding on diffusion-weighted MRI. Neuroradiology 2002; 44: 830-833

21 Peraud A, Illner A, Rutka JT. Intraventricular congenital lesions and colloid cysts. Neurosurgery clinics of North America 2003; 14: 607-619

22 Ducruet AF, Grobelny BT, Zacharia BE et al. The surgical management of chronic subdural hematoma. Neurosurgical review 2012; 35: $155-$ 169; discussion 169

23 Poels MM, Ikram MA, van der Lugt A et al. Incidence of cerebral microbleeds in the general population: the Rotterdam Scan Study. Stroke: a journal of cerebral circulation 2011; 42: 656-661

24 Schrag M, Greer DM. Clinical associations of cerebral microbleeds on magnetic resonance neuroimaging. Journal of stroke and cerebrovascular diseases: the official journal of National Stroke Association 2014; 23: 2489-2497

25 Mok V, Kim JS. Prevention and Management of Cerebral Small Vessel Disease. Journal of stroke 2015; 17: 111-122 
26 Debette S, Markus HS. The clinical importance of white matter hyperintensities on brain magnetic resonance imaging: systematic review and meta-analysis. BMJ (Clinical research ed) 2010; 341: c3666

27 Pantoni L, Fierini F, Poggesi A. Impact of cerebral white matter changes on functionality in older adults: An overview of the LADIS Study results and future directions. Geriatrics \& gerontology international 2015; $15: 10-16$

28 Dufouil C, Chalmers J, Coskun O et al. Effects of blood pressure lowering on cerebral white matter hyperintensities in patients with stroke: the PROGRESS (Perindopril Protection Against Recurrent Stroke Study) Magnetic Resonance Imaging Substudy. Circulation 2005; 112: 1644-1650

29 Okuda DT, Mowry EM, Beheshtian A et al. Incidental MRI anomalies suggestive of multiple sclerosis: the radiologically isolated syndrome. Neurology 2009; 72: 800-805

30 Forslin $Y$, Granberg T, Jumah AA et al. Incidence of Radiologically Isolated Syndrome: A Population-Based Study. American journal of neuroradiology 2016; DOI: 10.3174/ajnr.A4660

31 Okuda DT, Siva A, Kantarci 0 et al. Radiologically isolated syndrome: 5-year risk for an initial clinical event. PloS one 2014; 9: e90509

32 Granberg T, Martola J, Kristoffersen-Wiberg $M$ et al. Radiologically isolated syndrome-incidental magnetic resonance imaging findings suggestive of multiple sclerosis, a systematic review. Multiple sclerosis (Houndmills, Basingstoke, England) 2013; 19: 271 - 280

33 Holmstedt CA, Turan TN, Chimowitz MI. Atherosclerotic intracranial arterial stenosis: risk factors, diagnosis, and treatment. The Lancet Neurology 2013; 12: $1106-1114$

34 Zaidat OO, Castonguay AC, Nguyen TN et al. Impact of SAMMPRIS on the future of intracranial atherosclerotic disease management: polling results from the ICAD symposium at the International Stroke Conference. Journal of neurointerventional surgery 2014; 6: 225-230

35 Ryu WS, Park SS, Kim YS et al. Long-term natural history of intracranial arterial stenosis: an MRA follow-up study. Cerebrovascular diseases (Basel, Switzerland) 2014; 38: 290-296

36 Saini M, Suministrado MS, Hilal S et al. Prevalence and Risk Factors of Acute Incidental Infarcts. Stroke: a journal of cerebral circulation 2015; 46: $2722-2727$

37 Saini M, Ikram K, Hilal S et al. Silent stroke: not listened to rather than silent. Stroke: a journal of cerebral circulation 2012; 43: 3102 - 3104
38 Sailer AM, Wagemans BA, Nelemans PJ et al. Diagnosing intracranial aneurysms with MR angiography: systematic review and meta-analysis. Stroke: a journal of cerebral circulation 2014; 45: 119-126

39 Chalouhi N, Dumont AS, Randazzo C et al. Management of incidentally discovered intracranial vascular abnormalities. Neurosurgical focus 2011; 31: E1

40 Deutsche Gesellschaft für Neurologie. AWMF-Leitlinie Unrupturierte intrakranielle Aneurysmen. www.awmf.org/uploads/tx_szleitlinien/ 030-0301_S1_Unruptierte_intrakranielle_Aneurysmen_2012_verlaengert.pdf

41 Greving JP, Wermer MJ, Brown RD Jr et al. Development of the PHASES score for prediction of risk of rupture of intracranial aneurysms: a pooled analysis of six prospective cohort studies. The Lancet Neurology 2014; 13: 59-66

42 Josephson CB, Leach JP, Duncan $R$ et al. Seizure risk from cavernous or arteriovenous malformations: prospective population-based study. Neurology 2011; 76: 1548 - 1554

43 Ruiz DS, Yilmaz H, Gailloud P. Cerebral developmental venous anomalies: current concepts. Annals of neurology 2009; 66: 271 - 283

44 Gross BA, Puri AS, Popp AJ et al. Cerebral capillary telangiectasias: a meta-analysis and review of the literature. Neurosurgical review 2013; 36: 187-193; discussion 194

45 Barreau X, Marnat G, Gariel F et al. Intracranial arteriovenous malformations. Diagnostic and interventional imaging 2014; 95: 1175-1186

46 Spetzler RF, Ponce FA. A 3-tier classification of cerebral arteriovenous malformations. Clinical article. Journal of neurosurgery 2011; 114: $842-849$

47 Mohr JP, Parides MK, Stapf $C$ et al. Medical management with or without interventional therapy for unruptured brain arteriovenous malformations (ARUBA): a multicentre, non-blinded, randomised trial. Lancet (London, England) 2014; 383: 614-621

48 Chamoun R, Krisht KM, Couldwell WT. Incidental meningiomas. Neurosurgical focus 2011; 31: E19

49 Shah AH, Madhavan K, Sastry A et al. Managing intracranial incidental findings suggestive of low-grade glioma: learning from experience. World neurosurgery 2013; 80: e75-e77

50 Freda PU, Beckers AM, Katznelson L et al. Pituitary incidentaloma: an endocrine society clinical practice guideline. The Journal of clinical endocrinology and metabolism 2011; 96: 894-904 